\title{
ORIGINAL RESEARCH \\ Dampening of Blood-Flow Pulsatility along the Carotid Siphon: Does Form Follow Function?
}

T. Schubert
F. Santini
A.F. Stalder
J. Bock
S. Meckel
L. Bonati
M. Markl
S. Wetzel
BACKGROUND AND PURPOSE: The tortuous distal part of the ICA may have an attenuating effect on pulsatile arterial flow. We investigated local arterial blood flow patterns in the ICA proximal and distal to the carotid siphon to detect quantitative waveform changes.

\begin{abstract}
MATERIALS AND METHODS: Arterial flow patterns were analyzed by using flow-sensitized 4D PC MR imaging (time-resolved 3D PCMR) at 3T in 17 healthy volunteers. Time-resolved blood flow velocities were extracted from the source data at the C4 and C7 segments of the ICA. PI, RI, and PA were calculated by using time-resolved flow volume. A linear mixed-effects model was applied to compare values at $\mathrm{C} 4$ and $\mathrm{C} 7$. Furthermore, 3D blood flow visualization was performed for all 34 ICAs.
\end{abstract}

RESULTS: PI, RI, and PA were significantly lower at the distal C7 segment compared with the proximal C4 segment of the ICA $(P<.0001)$. Helical flow patterns were observed in 5 ICAs of 4 subjects.

CONCLUSIONS: Arterial flow patterns showed a significant reduction in PI, RI, and PA when compared distal to proximal to the carotid siphon. The observed attenuation of flow pulsatility is most likely related to the contorted shape of the distal ICA and may bear a protective effect for downstream cerebral vasculature.

ABBREVIATIONS: CCA $=$ common carotid artery; ICA $=$ internal carotid artery; $P A=$ peak acceleration of blood; $\mathrm{PCMR}=$ phase-contrast $\mathrm{MR}$ imaging; $\mathrm{PI}=$ pulsatility index; $\mathrm{RI}=$ resistance index
$\mathbf{A}_{\mathrm{i} \text { p }}^{\mathrm{p}}$ precise characterization of normal arterial flow waveforms in the carotid arteries is important because the normal waveform and its alterations may contribute to our understanding of cerebrovascular disease. The evaluation of local alterations of the arterial waveform are widely used for the diagnosis of arterial stenoses. ${ }^{1}$ However, little is known about physiologic and pathologic waveform changes while traversing the arterial tree from the heart to the peripheric vessels. To identify normal blood flow profiles, several studies focused on the characterization of archetypal flow waveforms in the carotid artery tree of healthy volunteers of different ages, ${ }^{2-5}$ by using either Doppler sonography or 2D phase-contrast MR imaging.

Most of these studies provided detailed qualitative and quantitative descriptions of the normal arterial flow waveform at a single location per vessel. One publication, however, analyzed flow waveforms at different sites along the ICA to depict possible waveform changes. ${ }^{6}$

In the present study, we applied flow-sensitive 4D PCMR imaging (time-resolved 3D PCMR) to assess carotid artery flow. In contrast to most 2D PCMR applications that use unidirectional velocity encoding, 4D PCMR allows velocity encoding multidirectionally in the acquired $3 \mathrm{D}$ data volume. A further advantage is the ability to measure blood flow at different sites within the same cardiac cycles. Compared with Doppler sonography, all MR imaging applications are able to

Received June 27, 2010; accepted after revision October 15.

From the Departments of Radiology (T.S., S.W.) and Neurology (L.B.), University Hospital Basle, Basle, Switzerland; Department of Radiology (F.S.), Medical Physics, University Hospital Basle, Basle, Switzerland; Department of Radiology (A.F.S., J.B., M.M.), Medical Physics, University Hospital Freiburg, Freiburg, Germany; Department of Neuroradiology (S.M.), University Hospital Freiburg, Freiburg, Germany; Department of Neuroradiology (S.W.), Swiss Neuro Institute Zurich, Hirslanden Clinic, Zurich, Switzerland; and Department of Radiology (A.F.S.), Xuanwu Hospital of Capital Medical University, Beijing, China.

Please address correspondence to Tilman Schubert, MD, Department of Radiology, University Hospital Basle, Basle, Switzerland; email: TSchubert@uhbs.ch

DOI 10.3174/ajnr.A2426 measure flow users independently and without anatomic restrictions.

With 4D PCMR being particularly capable of capturing blood flow in geometrically complex vessel sections, we measured carotid artery blood flow along a tortuous section with little interindividual variance, the carotid siphon. We hypothesized that the carotid siphon may have an attenuating effect on blood flow pulsatility. To prove this hypothesis, we measured arterial blood flow proximal and distal to the carotid siphon in healthy volunteers. To quantify arterial flow waveforms, PI, RI, and PA were calculated. PI and RI are commonly used parameters for waveform characterization and quantification of pulsatility. ${ }^{7} \mathrm{PA}$, a valuable index of peripheral flow pulsatility, though less common, can add explanatory power in addition to the PI and RI, depicting the ascending slope of the flow wave. 8,9

In addition to quantitative flow waveform analysis, blood flow patterns within the region of interest were visualized. Blood flow visualization may provide additional information about alterations in arterial blood flow patterns. Previously reported results demonstrated the potential of this technique for the assessment of normal and altered blood flow in the heart, the aorta, and the common carotid artery. ${ }^{10-17}$

\section{Materials and Methods}

\section{Study Population}

Thirty-four ICAs of 17 healthy volunteers (10 men, 7 women; median age, 22.5 years; range, 19-32 years) were investigated. For each vessel, blood flow patterns were visualized and time-resolved blood flow measurement was performed. The study was approved by the local ethics review committee, and written informed consent was obtained from all subjects.

\section{MR Imaging}

All examinations were performed on a 3T head system (Magnetom Allegra; Siemens, Erlangen, Germany) by using a standard head coil. 

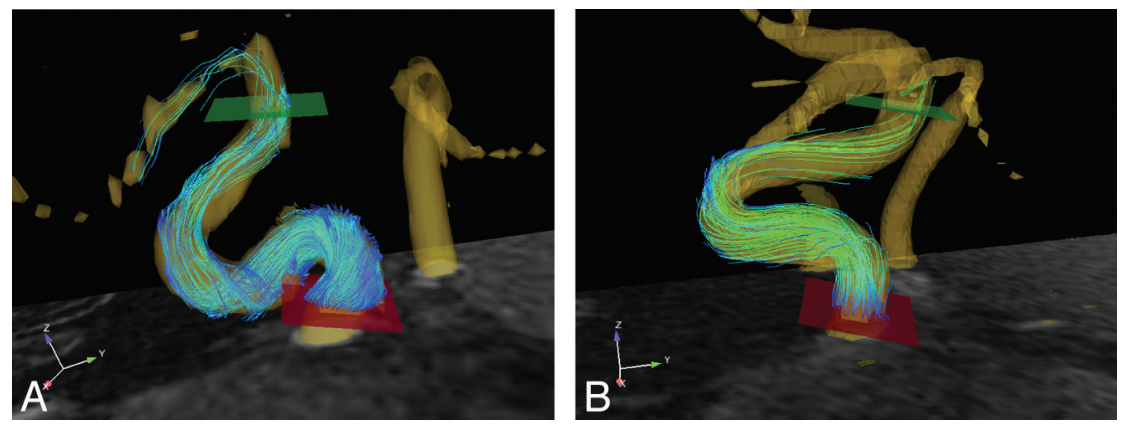

Fig 1. Placement of $2 \mathrm{D}$ planes for flow measurement at the $\mathrm{C} 4$ and $\mathrm{C7}$ segments in subjects with helical $(A)$ and laminar (B) carotid blood flow patterns
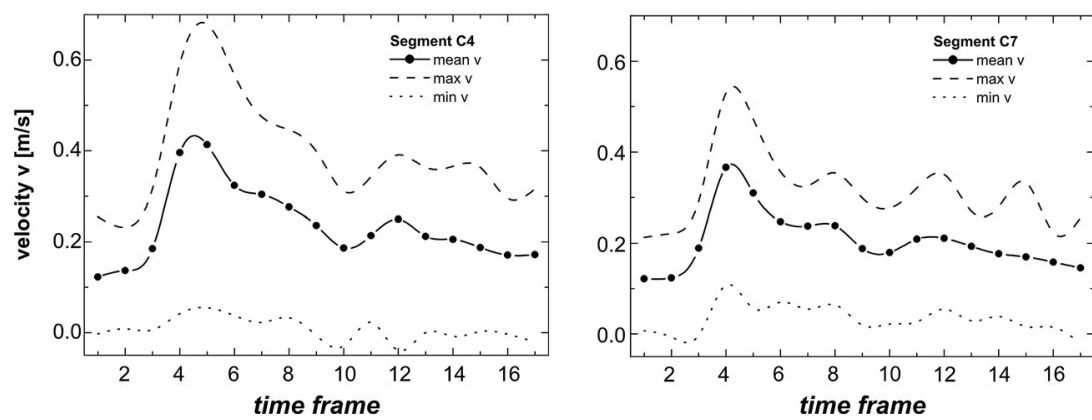

Fig 2. Velocity diagrams at the C4 (left) and C7 (right) segments of 1 individual carotid artery with peak acceleration values of $74 \mathrm{~mL} / \mathrm{s}^{2}$ at $\mathrm{C} 4$ and $34 \mathrm{~mL} / \mathrm{s}^{2}$ at C7.
Flow-sensitive 4D MR imaging consisted of a $k$-space segmented 3D radio-frequency-spoiled gradient-echo sequence with prospective electrocardiography gating and interleaved 3-directional velocity encoding. Data acquisition resulted in a series of $3 \mathrm{D}$ volumes representing $3 \mathrm{D}$ blood flow in consecutive timeframes within the cardiac cycle with a temporal resolution of $54.4-56.0 \mathrm{~ms} .{ }^{18}$ The total acquisition times ranged between 25 and 30 minutes, depending on the patient's individual heart rate (13-16 acquired cardiac phases). Further imaging parameters were the following: TR/TE, 6.8-7.0/4.0-4.4 ms; flip angle $\alpha, 15^{\circ}$; band width, $450 \mathrm{~Hz} /$ pixel. The velocity distributions for each voxel and time point within the cardiac cycle were reconstructed from data acquired over numerous cardiac cycles throughout the total acquisition period. The axial 3D slab was positioned to cover the carotid siphon with the C5 segment (center of the carotid siphon) equidistant to the upper and lower boundary of the acquired data volume $(220 \times 176 \mathrm{~mm}$ rectangular FOV $)$ with 28 sections per slab and a spatial resolution of $1.0 \times 0.7 \times 1.2 \mathrm{~mm}$. Images were reconstructed to a matrix of $320 \times 256$ by using zero-filling, resulting in a reconstructed resolution of $0.7 \times 0.7 \times 1.2 \mathrm{~mm}$.

\section{Postprocessing}

Automated postprocessing of 4D datasets by using an in-house software tool based on Matlab (MathWorks, Natick, Massachusetts) included noise-masking, antialiasing, and eddy current correction as previously described. ${ }^{14,19}$ For visualization of vascular geometry and spatially registered blood flow, a commercially available $3 \mathrm{D}$ visualization software package (EnSight; CEI, Apex, North Carolina) was used.

Flow measurement was performed by retrospectively placing $2 \mathrm{D}$ analysis planes transecting the arterial lumen perpendicular to the main axis of the ICA at segment locations C4 and C7 (Fig 1) (ie, proximal and distal to the carotid siphon). The time-resolved velocity data for all 3 spatial velocity components within the analysis planes were imported into an in-house analysis tool based on Matlab (MathWorks). ${ }^{20}$ Frame-wise interactive segmentation of the vessel lumen was performed on the magnitude images by using cubic B-spline smooth contours. ${ }^{21}$ On the basis of the segmented vessel lumen contour, we extracted peak flow (milliliters/second), minimal flow (milliliters/second), and peak acceleration (milliliters/second squared) from the flow velocity time curves (Fig 2).

\section{Outcome Measures}

For quantification of pulsatility, PI, RI, and PA were applied.

The PI and RI were calculated according to the following formulas by using the volume flow rate ${ }^{22}$ :

Pulsatility Index:

$$
P I=\frac{F \max -F \min }{F \text { mean }}
$$

Resistance Index:

$$
R I=\frac{F \max -F \text { enddiast }}{F \min },
$$

where $F$ max indicates maximum blood volume flow rate; $F$ min, minimum blood volume flow rate; $F$ mean, mean blood volume flow rate; and $F$ enddiast, blood volume flow rate at the end of diastole.

The volumetric flow was interpolated with time on the basis of a cubic B-spline model, ${ }^{23}$ which creates an analytic and continuous version of the volumetric flow on the basis of a least curvature property (ie, minimum second-order differentiation). This model allows a temporal interpolation between measurement points and, at the same time, provides a continuous differentiation of the volumetric flow. On the basis of this, it was possible to derive the flow acceleration and calculate the peak flow acceleration.

\section{Visualization}

As a qualitative analysis, blood flow visualization was performed. Blood flow within every 3D data volume was visualized by using time-resolved 3D particle traces resembling the spatial and temporal distribution of virtual massless particles tracing the measured 3directional blood velocity vector fields. Virtual particles were released at the start of systole and originated from 100 grid points equally distributed within emitter planes in the arterial lumen. Using an isosurface representation of vessel contours displaying the sum of squares of velocities in all directions over the cardiac cycles for ana- 
Table 1: Estimates and results of the likelihood ratio tests for the fixed effects of the linear mixed-effects model

\begin{tabular}{lcccc}
\hline End Point & Contrast & Estimate & $\chi^{2}(1)$ & $P$ Value \\
\hline PI & Distal-proximal & -0.19 & 37.8 & $<.0001$ \\
RI & Distal-proximal & -0.08 & 27.5 & $<.0001$ \\
PA & Distal-proximal & -0.03 & 31.2 & $<.0001$ \\
\hline
\end{tabular}

tomic orientation, we positioned emitter planes at the $\mathrm{C} 4$ and $\mathrm{C} 7$ segments of the ICA on both sides. The resulting 3D particle traces were color-coded according to the local velocity and animated to resemble in vivo $3 \mathrm{D}$ blood flow.

The $3 \mathrm{D}$ blood flow characteristics were evaluated for the presence of helical flow by consensus reading (T.S., F.S., S.W.) by using information provided by time-resolved 3D images representing 1 cardiac cycle. The ICA was visualized in axial and longitudinal views. $3 \mathrm{D}$ particle traces were analyzed frame by frame for a rotational component.

"Helical flow" was defined as a rotation of at least $180^{\circ}$ in a longitudinal vessel direction assessed by manually following the particle traces.

\section{Statistical Analysis}

The 3 end points, PI, RA, and PA, were calculated for the left and right ICAs at segment locations C4 and C7 for all 17 volunteers. For each end point, a linear mixed-effects model was applied with segment location as a mixed effect and volunteer and side (nested within volunteer) as random effects for which separate intercepts were used. The significance of mixed effects was assessed with likelihood ratio tests by comparing the original model and a model reduced by the mixed effect (segment location). The level of significance was set at $P<.05$.

\section{Results}

Mean PI decreased by $17.4 \%$ (range, $-52 \%$ to $+5 \%$ ); RI, by $14.5 \%$ (range, $-39 \%$ to $+14 \%$ ); and $\mathrm{PA}$, by $38.1 \%$ (range, $-61 \%$ to $+37 \%)$. According to the results of the mixed-effects models, all 3 end points (PI, RI, and PA) were significantly reduced if distal and proximal locations were compared $(P<$ .0001, Table 1 and Fig 3). The estimated variances between volunteers were $0.0111,0.00746$, and 0.000775 for PI, RI, and $\mathrm{PA}$, respectively. The estimated variances between left and right side measurements were negligible.

Flow visualization revealed helical flow patterns in $5 \mathrm{ca}-$ rotid arteries of 4 subjects (Fig 4 ).

\section{Discussion}

The main finding of the present study is a highly significant decrease in arterial pulsatility from proximal to distal to the carotid siphon. As far as we know, this result has not been previously noted. The distinct reduction in pulsatility values appears along a very short vessel section, which leads to the assumption that the decrease in pulsatility from the $\mathrm{C} 4$ to the C7 segment is explained by specific properties of the carotid siphon. The examined part of the ICA has a tortuous appearance with, among other curvatures, 1 going beyond a $180^{\circ}$ bend with astounding interindividual uniformity.

That characteristic shape may be an explanation of the attenuation of blood flow pulsatility along the carotid siphonthat is, in contrast to a straight vessel, a fluid running through a curved vessel generates a centrifugal force, ${ }^{24}$ leading to an energy transfer from the current to the vessel wall. With regard to a physiologic pulsatile current, energy transfer from the current to the vessel wall takes place at peak systolic velocity and vice versa in the diastolic phase. The elastic vessel wall can react on the applied force with distension during systole and retraction during diastole, which may attenuate downstream flow pulsatility appropriate to a Windkessel-reservoir.

The results of the present study are plausible, for the following reasons: 1) The acquired values were consistent with low intersubject variability, 2) all except 1 subject showed a decrease in PI, RI, and PA, and 3) each evaluated parameter, PI, RI, and PA, showed a statistically highly significant decrease from proximal to distal (Table 2).

In contrast to the present findings, flow pulsatility decreases along the course of the carotid arteries from proximal, CCA, to distal, ICA. ${ }^{25}$ In contrast, detailed assessment of hemodynamic parameters at different sites of the ICA has received relatively little attention so far. However, in a recent study, Gwilliam et $\mathrm{al}^{6}$ assessed arterial pulsatility in a collection of healthy volunteers in the CCA and the ICA in both its proximal and distal course with 2D PCMR. A high reduction of pulsatility was found in the ICA immediately after the bifurcation compared with the CCA. However, when measurements in the proximal and distal ICA (distal to the carotid siphon) were compared, overall only a slight reduction in pulsatility was found, which was not statistically significant.

The discrepancy between the findings of Gwilliam et $\mathrm{al}^{6}$ and our own results is most likely related to the underlying MR imaging technique for flow measurement. Along vessels without long straight sections, like the segments C3-C7 of the ICA, 2D PCMR with unidirectional velocity encoding can lead to an underestimation of peak flow values. In those vessel sections, it is likely that a significant velocity component diverges from the longitudinal vessel axis. In addition, 5-mm section thickness, which is commonly used in 2D PCMR, may be inappropriate. We, therefore, assume that the underestimation of peak flow velocities in 2D PCMR appears disproportionally in curved vessel sections.

Furthermore, optimized placement of measurement planes by using 2D PCMR in tortuous vessels is complex and has to be done prospectively. Even slight deviations of the measurement plane can result in a significant difference of measured and true peak velocity. Retrospectively detected placement errors cannot be corrected.

These limitations of 2D PCMR may explain the results of Gwilliam et al, ${ }^{6}$ in which no significant reduction in pulsatility along the carotid siphon was found.

Using 3D PCMR with multidirectional velocity encoding, one can avoid underestimation of peak flow values due to complex 3D flow patterns. Furthermore, optimal placement of measurement planes in the volume of interest is performed retrospectively, and vessel contours can be magnified at will. The higher spatial resolution of 3D PCMR compared with 2D PCMR (here, $1.0 \times 0.7 \times 1.2 \mathrm{~mm}$ versus $1 \times 1 \times 5 \mathrm{~mm}$ ) reduces intravoxel dephasing effects; this result allows a more detailed measurement of peak flow velocities and effectively covers multidirectional flow. Further advantages include the ability to measure flow at different sites within the acquired data volume over the same cardiac cycles. The lower temporal resolution of $4 \mathrm{D}$ PCMR, however, may lead to an underesti- 


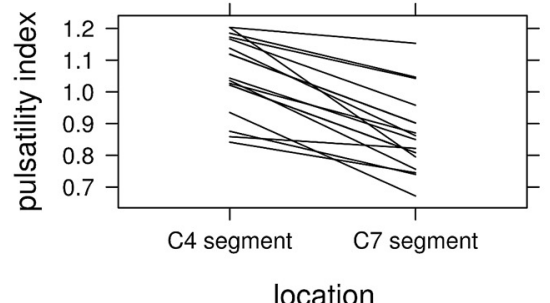

location

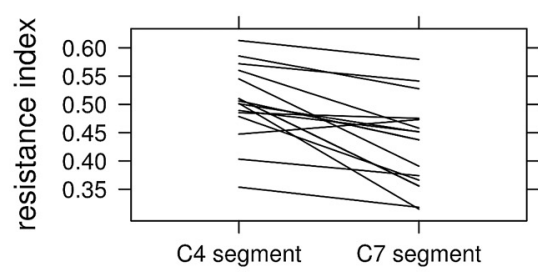

location

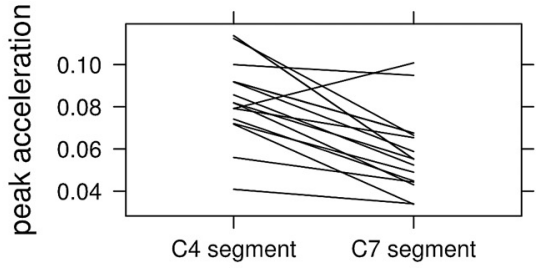

location

Fig 3. PI (top), RI (middle), and PA (bottom) of the 17 volunteers proximally (at segment C4) and distally (segment C7) averaged over the left and right ICAs.
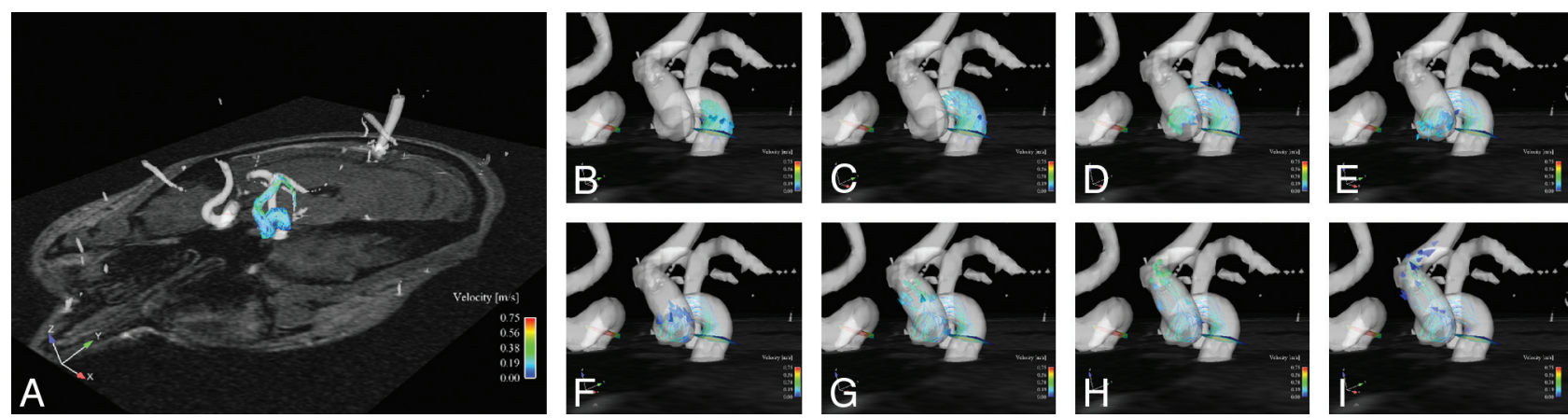

Fig 4. Time-resolved blood flow visualization in a subject with helical blood flow in the left ICA.

Table 2: Mean values and the standard error of PI, RI, and PA proximal (C4) and distal (C7) to the carotid siphon ${ }^{a}$

\begin{tabular}{lccc}
\hline End Point & Location & Mean Value & $\begin{array}{c}\text { Standard } \\
\text { Error }\end{array}$ \\
\hline PI & Proximal (C4), & 1.0568 & 0.02425 \\
& distal (C7) & 0.8666 & 0.02576 \\
RI & Proximal (C4), & 0.5039 & 0.01204 \\
& distal (C7) & 0.4299 & 0.01409 \\
PA & Proximal (C4), & 0.0846 & 0.00375 \\
& distal (C7) & 0.0581 & 0.00364 \\
\hline
\end{tabular}

a The standard error is defined as the SD of the sampling distribution associated with the estimation method.

mation of peak flow values. In contrast to 2D PCMR, this effect appears uniformly along the vessel independent of its geometric complexity.

However, the study of Gwilliam et $\mathrm{al}^{6}$ and ours are not fully comparable due to the different objectives and statistical approaches. In the present study, a general linear model was applied for a selective systematic comparison of pulse-wave characteristics at 2 locations. In contrast, the primary goal of the study of Gwilliam et al was to provide characteristic volumetric flow rate waveforms at certain locations in the brainsupplying arteries. Pulsatility indices at these locations were additionally compared by using the Student $t$ test; no information was provided as to whether paired or unpaired $t$ tests were applied.

Because the present study was designed to analyze flow waveforms along the carotid siphon, artifacts due to gradientfield inhomogeneities were unlikely because the regions of interest were properly centered in the acquired 3D dataset. The accuracy of the applied 4D PCMR method has been confirmed by using $2 \mathrm{D}$ cine PCMR. ${ }^{26}$

Physiologically, the measured decrease in arterial pulsatility along the carotid siphon may be meaningful for the cerebral circulation. The brain exhibits a low-resistance capillary bed ${ }^{27}$ for which increased arterial pulsatility may be harmful.
In patients with type 2 diabetes mellitus, increased arterial pulsatility correlates with a higher degree of cerebral microangiopathy. ${ }^{28-30}$ Another example is a recent study by Bateman et al, ${ }^{31}$ which showed that early vascular dementia is characterized by increased blood flow pulsation.

However, the clinical significance of the dampening function of the carotid siphon with possible changes in the C4/C7 pulsatility ratio must be further evaluated in patient studies.

\section{Blood Flow Visualization}

As a qualitative analysis, time-resolved 3D blood flow was visualized in each ICA. This analysis was performed because the visualization was integral to optimally placing measurement planes. A further purpose was the evaluation of helical flow patterns that were observed in the ICA and CCA in a previously published feasibility study of $4 \mathrm{D}$ PCMR. ${ }^{15,21}$ In the present study, however, helical flow patterns were observed in only a small number of arteries (5 of 34 ICAs, Fig 4), and a statistically relevant analysis assessing the effect of helical flow on arterial pulsatility could not be performed. This low number of helical flow patterns may be related, on one hand, to the young age of the volunteers and, on the other hand, to the scanning length of the applied method. In aged subjects, elongation of the ICA may lead to a higher fraction of helical flow patterns in the ICA. ${ }^{32}$ However, we believe that the relationship between vessel geometrics, resulting geometric flow patterns, and arterial pulsatility deserves further attention.

$4 \mathrm{D}$ PCMR is a promising tool for gaining a better understanding of local hemodynamics under physiologic and pathologic conditions. Future improvements of spatiotemporal resolution will facilitate flow visualization in high-grade stenoses and vessels of small diameters.

\section{Methodologic Limitations}

Currently, the limitations of the methodology are mainly related to the spatial and temporal resolution and the total scan- 
ning time requirement. With the given voxel size of $1.0 \times$ $0.7 \times 1.2 \mathrm{~mm}$ and a cross-section of the ICA of approximately $12-16 \mathrm{~mm}^{2}$, the spatial resolution is appropriate. However, in vessels of significantly smaller diameter, partial volume effects (ie, voxels containing fractions of arterial lumen as well as surrounding tissue) need to be considered and may limit the accuracy of the measured velocities. Furthermore, the temporal resolution of approximately $55 \mathrm{~ms}$ was relatively low, which might lead to an underestimation of peak velocities due to low-pass filtering, which may affect the derived PIs and RIs, which rely on the differences between peak and minimum velocities in the cardiac cycle. Thus, both spatial and temporal averaging likely contributed to the lower peak velocities observed in a preliminary assessment in comparison with transcranial Doppler sonography $^{18}$; this finding is consistent with previous reports that compared peak velocities from PCMR and Doppler sonography. ${ }^{33,34}$ Because of the limited spatiotemporal resolution and for calculating flow acceleration, a cubic B-spline model was used in this study. Due to the minimal second-order differentiation property of cubic B-spline, it is particularly adapted to fluids, ${ }^{20}$ which follow the continuity laws (conservation of mass and momentum law). ${ }^{23}$ The B-spline model did not affect the measured data points but allowed derivation of interpolated data points up to a secondorder differentiation (thus allowing calculation of flow acceleration). Nevertheless, this model cannot fully replace measured data, and the discrete nature of the acquired data might be responsible for some errors in the calculated parameters. In particular, limited temporal resolution might be responsible for a certain underestimation of flow acceleration.

A disadvantage of flow-sensitive 4D MR imaging is related to the rather long scanning times. To shorten overall acquisition times, the application of new spatiotemporal imaging acceleration techniques (eg, k-t BLAST, k-t GRAPPA) is promising because redundancies in 2 spatial encoding dimensions and the temporal dimension can be used to speed up data acquisition. ${ }^{35,36}$ In addition, new methods based on the combination of PCMR and fast-sampling strategies (eg, radial imaging with $3 \mathrm{D}$ phase-contrast vastly undersampled isotropic projection reconstruction) have been reported and are highly promising for further reduction in total scanning time. ${ }^{37}$

The long scanning time may lead to motion artifacts, which can affect the vessel boundary due to partial volume effects, whereas the central voxels are much less likely to be affected. This may lead to an abruption of the outer particle traces.

Further limitations of the study involve the assessment of ICA blood flow patterns only in young healthy individuals, and hemodynamic properties evaluated only along the curved distal part of the ICA. However, a characterization of blood flow patterns with 4D PCMR covering the full length of the ICA is planned.

Nonetheless, these limitations do not undermine the legitimacy of the main result but merely point to a number of possible improvements for further research in this area.

\section{Outlook}

The next steps for the application of the 4D technique should include the assessment of the age effect on arterial flow patterns. Furthermore, patient cohort studies are necessary to assess pulsatility values for specific diseases. If spatial and temporal resolution of 4D PCMR can be effectively improved and shorter measurement times can be obtained, it is likely that this technique will find a more widespread application.

\section{Acknowledgments}

We thank the Clinical Trial Unit, Department of Biostatistics, Basle University, Switzerland, for statistical analysis and Philip Ebert, PhD, Department of Logics and Metaphysics, Stirling University, United Kingdom, for proofreading.

\section{References}

1. Kim ES, Thompson M, Nacion KM, et al. Radiologic importance of a highresistive vertebral artery Doppler waveform on carotid duplex ultrasonography. J Ultrasound Med 2010;29:1161-65

2. Ford MD, Alperin N, Lee $\mathrm{SH}$, et al. Characterization of volumetric flow rate waveforms in the normal internal carotid and vertebral arteries. Physiol Meas 2005;26:477-88. Epub 2005 Apr 29

3. Hoi Y, Wasserman BA, Xie YJ, et al. Characterization of volumetric flow rate waveforms at the carotid bifurcations of older adults. Physiol Meas 2010;31: 291-302. Epub 2010 Jan 20

4. Holdsworth DW, Norley CJ, Frayne R, et al. Characterization of common carotid artery blood-flow waveforms in normal human subjects. Physiol Meas 1999;20:219-40

5. Scheel $\mathrm{P}$, Ruge $\mathrm{C}$, Schoning $\mathrm{M}$. Flow velocity and flow volume measurements in the extracranial carotid and vertebral arteries in healthy adults: reference data and the effects of age. Ultrasound Med Biol 2000;26:1261-66

6. Gwilliam MN, Hoggard N, Capener D, et al. MR derived volumetric flow rate waveforms at locations within the common carotid, internal carotid, and basilar arteries. J Cereb Blood Flow Metab 2009;29:1975-82

7. Gosling RG, King DH, Woodcock JP. Transcutaneous measurements of arterial blood-velocity by ultrasound. J Ultrasonics USI Conf Papers 1969;16-23

8. Chemla D, Demolis P, Thyrault M, et al. Blood flow acceleration in the carotid and brachial arteries of healthy volunteers: respective contributions of cardiac performance and local resistance. Fundam Clin Pharmacol 1996;10: 393-99

9. Targett RC, Levy B, Bardou A, et al. Simultaneous Doppler blood velocity measurements from aorta and radial artery in normal human subjects. Cardiovasc Res 1985;19:394-99

10. Bogren HG, Buonocore $\mathrm{MH}$. 4D magnetic resonance velocity mapping of blood flow patterns in the aorta in young vs. elderly normal subjects. J Magn Reson Imaging 1999;10:861-69

11. Bogren HG, Mohiaddin RH, Kilner PJ, et al. Blood flow patterns in the thoracic aorta studied with three-directional MR velocity mapping: the effects of age and coronary artery disease. J Magn Reson Imaging 1997;7:784-93

12. Bogren HG, Mohiaddin RH, Yang GZ, et al. Magnetic resonance velocity vector mapping of blood flow in thoracic aortic aneurysms and grafts.J Thorac Cardiovasc Surg 1995;110:704-14

13. Buonocore $\mathrm{MH}$. Algorithms for improving calculated streamlines in 3-D phase contrast angiography. Magn Reson Med 1994;31:22-30

14. Buonocore $\mathrm{MH}$. Visualizing blood flow patterns using streamlines, arrows, and particle paths. Magn Reson Med 1998;40:210-26

15. Harloff A, Albrecht F, Spreer J, et al. 3D blood flow characteristics in the carotid artery bifurcation assessed by flow-sensitive 4D MRI at 3T. Magn Reson Med 2009;61:65-74

16. Kilner PJ, Gatehouse PD, Firmin DN. Flow measurement by magnetic resonance: a unique asset worth optimising. J Cardiovasc Magn Reson 2007; 9:723-28

17. Wigstrom L, Ebbers T, Fyrenius A, et al. Particle trace visualization of intracardiac flow using time-resolved 3D phase contrast MRI. Magn Reson Med 1999;41:793-99

18. Wetzel S, Meckel S, Frydrychowicz A, et al. In vivo assessment and visualization of intracranial arterial hemodynamics with flow-sensitized 4D MR imaging at 3T. AJNR Am J Neuroradiol 2007;28:433-38

19. Walker PG, Cranney GB, Scheidegger MB, et al. Semiautomated method for noise reduction and background phase error correction in MR phase velocity data. J Magn Reson Imaging 1993;3:521-30

20. Stalder AF, Russe MF, Frydrychowicz A, et al. Quantitative 2D and 3D phase contrast MRI: optimized analysis of blood flow and vessel wall parameters. Magn Reson Med 2008;60:1218-31

21. Meckel S, Stalder AF, Santini F, et al. In vivo visualization and analysis of 3-D hemodynamics in cerebral aneurysms with flow-sensitized 4-D MR imaging at 3 T. Neuroradiology 2008;50:473-84

22. Beldi G, Bosshard A, Hess OM, et al. Transit time flow measurement: experimental validation and comparison of three different systems. Ann Thorac Surg 2000;70:212-17 
23. Unser M. Splines: a perfect fit for signal and image processing. IEEE Signal Processing Magazine 1999;16:22-38

24. Kundu PK, Cohen TM. Fluid Mechanics. San Diego, California: Elsevier Academic Press; 2004:77-128

25. Nichols WM, O'Rourke MF. McDonald's Blood Flow in Arteries: Theoretical, Experimental and Clinical Principles. 5th ed. London: Hodder Arnold; 2005

26. Markl M, Chan FP, Alley MT, et al. Time-resolved three-dimensional phasecontrast MRI. J Magn Reson Imaging 2003;17:499-506

27. Chavhan GB, Parra DA, Mann A, et al. Normal Doppler spectral waveforms of major pediatric vessels: specific patterns. Radiographics 2008;28:691-706

28. Galler A, Heitmann A, Siekmeyer W, et al. Increased arterial stiffness in children and adolescents with type 1 diabetes: no association between arterial stiffness and serum levels of adiponectin. Pediatr Diabetes 2010;11:38-46. Epub 2009 May 28

29. Lee KY, Sohn YH, Baik JS, et al. Arterial pulsatility as an index of cerebral microangiopathy in diabetes. Stroke 2000;31:1111-15

30. Matsumoto K, Sera Y, Nakamura H, et al. Correlation between common carotid arterial wall thickness and ischemic stroke in patients with type 2 diabetes mellitus. Metabolism 2002;51:244-47
31. Bateman GA, Levi CR, Schofield P, et al. The venous manifestations of pulse wave encephalopathy: windkessel dysfunction in normal aging and senile dementia. Neuroradiology 2008;50:491-97. Epub 2008 Apr 1

32. Lam RC, Lin SC, DeRubertis B, et al. The impact of increasing age on anatomic factors affecting carotid angioplasty and stenting. J Vasc Surg 2007;45:875-80

33. Karwatowski SP, Brecker SI, Yang GZ, et al. Mitral valve flow measured with cine MR velocity mapping in patients with ischemic heart disease: comparison with Doppler echocardiography. J Magn Reson Imaging 1995;5:89-92

34. Oktar SO, Yucel C, Karaosmanoglu D, et al. Blood-flow volume quantification in internal carotid and vertebral arteries: comparison of 3 different ultrasound techniques with phase-contrast MR imaging. AJNR Am J Neuroradiol 2006;27:363-69

35. Baltes C, Kozerke S, Hansen MS, et al. Accelerating cine phase-contrast flow measurements using k-t BLAST and k-t SENSE. Magn Reson Med 2005;54: $1430-38$

36. Jung $\mathrm{B}$, Honal $\mathrm{M}$, Ullmann $\mathrm{P}$, et al. Highly k-t-space-accelerated phasecontrast MRI. Magn Reson Med 2008;60:1169-77

37. Johnson KM, Lum DP, Turski PA, et al. Improved 3D phase contrast MRI with off-resonance corrected dual echo VIPR. Magn Reson Med 2008;60:1329-36 